\title{
Bacterial Lipoproteins Can Disseminate from the Periphery to Inflame the Brain
}

\author{
Diana Londoño and Diego Cadavid \\ From the Department of Neurology and Neuroscience and Center \\ for Emerging Pathogens at University of Medicine and Dentistry \\ of New Jersey, New Jersey Medical School, Newark, New Jersey; \\ and Center for Immunology and Inflammatory Diseases at \\ Massachusetts General Hospital, Boston, Massachusetts
}

The current view is that bacteria need to enter the brain to cause inflammation. However, in mice infected with the spirochete Borrelia turicatae, we observed widespread cerebral inflammation despite a paucity of spirochetes in the brain parenchyma at times of high bacteremia. Here we studied the possibility that bacterial lipoproteins may be capable of disseminating from the periphery across the bloodbrain barrier to inflame the brain. For this we injected normal and infected mice intraperitoneally with lanthanide-labeled variable outer membrane lipoproteins of $B$. turicatae and measured their localization in blood, various peripheral organs, and whole and capillary-depleted brain protein extracts at various times. Lanthanide-labeled nonlipidated lipoproteins of $B$. turicatae and mouse albumin were used as controls. Brain inflammation was measured by TaqMan RT-PCR amplification of genes known to be upregulated in response to borrelial infection. The results showed that the two lipoproteins we studied, LVsp1 and LVsp2, were capable of inflaming the brain after intraperitoneal injection to different degrees: LVsp1 was better than LVsp2 and Bt1 spirochetes at moving from blood to brain. The dissemination of LVsp1 from the periphery to the brain occurred under normal conditions and significantly increased with infection. In contrast, LVsp2 disseminated better to peripheral organs. We conclude that some bacterial lipoproteins can disseminate from the periphery to inflame the brain. (Am J Pathol 2010, 176:2848-2857; DOI: 10.2353/ajpath.2010.091235)

The traditional belief is that bacteria need to cross the blood brain barrier (BBB) to cause brain inflammation. However, during studies in mice persistently infected with the relapsing fever spirochete Borrelia turicatae, we noticed wide- spread cerebral microgliosis that could not be explained by the presence of spirochetes in the brain parenchyma. ${ }^{1-4}$ Because cerebral microgliosis is much more pronounced during persistent than during intermittent bacteremia, ${ }^{4,5}$ the possibility was raised that movement of pro-inflammatory bacterial products from blood to brain rather than the bacteria themselves could be responsible. Release of pro-inflammatory bacterial products occurs from spirochetes both spontaneously ${ }^{6}$ and upon immune or antibiotic mediated lysis. ${ }^{7-9}$ Furthermore, release of outer membrane lipoproteins from spirochetes into the extracellular environment can occur independently of blebs. ${ }^{6}$

However, little is known about whether bacterial products can be released in vivo and disseminate into tissues like the brain to cause inflammation. ${ }^{10}$ Studies with lipopolysaccharide (LPS) from Gram-negative bacteria have shown that it does not cross the BBB but rather appears to cause brain inflammation by binding to its receptor, TLR4, on brain endothelial cells. ${ }^{11}$ Although borrelia spirochetes lack LPS, they do contain an abundance of potent pro-inflammatory outer membrane lipoproteins that share a common palmitate 3 Cysteine modification with many other bacterial species. ${ }^{12-16}$ Bacterial lipoproteins play prominent roles in disease pathogenesis. ${ }^{12}$ Many, if not all, of their biological activities are dependent on the lipid modification. ${ }^{15}$ The ability of eukaryotic cells to respond to lipoproteins correlates with expression of TLR2. ${ }^{17}$

Here we studied whether bacterial lipoproteins are capable of disseminating to the brain from the periphery. For this we injected mice intraperitoneally with lipidated outer membrane lipoproteins LVsp1 or LVsp2 of the relapsing fever

Supported by NIH grants R21 NS053997 and R21 NS057545-02 to D.C. Accepted for publication February 25, 2010.

This content is solely the responsibility of the authors and does not necessarily represent the official views of the National Institute of Neurological Disorders and Stroke or the National Institutes of Health.

D.C. is currently a full-time paid employee of Biogen Idec. His work on this article is not related to his employment in Biogen Idec.

Supplemental material for this article can be found on http://ajp. amjpathol.org.

Address reprint requests to Diego Cadavid, M.D., Center for Immunology and Inflammatory Diseases, Massachusetts General Hospital, 149 13th St, Room 8301, Charlestown, MA 02129. E-mail: dcadavid@ partners.org 
spirochete $B$. turicatae or with mouse albumin as a nonspecific control. We chose to compare LVsp1 with LVsp2 because they are the major outer membrane lipoproteins of isogenic serotypes that exhibit marked differences in neurotropism during infection. ${ }^{1,18}$ We also included nonlipidated recombinant $V s p 1$ ( $\mathrm{V} V \mathrm{sp} 1$ ) to investigate the role of the lipidation on bacterial lipoprotein dissemination. All proteins were labeled with Europium (Eu) or Samarium (Sm), two time-resolved fluorescence lanthanides that offer an alternative to isotopic labeling with high sensitivity and stability. ${ }^{19}$ Crossing of the BBB as opposed to localization within the cerebral microvasculature was studied by comparing perfused whole brain homogenates with perfused capillary-depleted and capillary-enriched brain homogenates. Inflammation was measured by TaqMan RT-PCR amplification of genes known to be up-regulated in response to borrelial infection. The results revealed that bacterial lipoproteins are capable of disseminating throughout the body to a different degree after local release. Equally important was the finding that LVsp1 to a greater extent than LVsp2, but not rVsp1, was capable of crossing the BBB from the circulation and inflaming the brain.

\section{Materials and Methods}

\section{Protein Labeling and Purification}

The following proteins were used in this study: (1) LVsp1 and LVsp2, the major outer membrane lipoproteins of serotypes 1 (Bt1) and 2 (Bt2), respectively, of the relapsing fever spirochete $B$. turicatae; they were prepared by detergent extraction from cultures of Bt1 or Bt2 spirochetes as before ${ }^{20}$; (2) rVsp1 was kindly provided by Wolfram Zuckert, PhD (University of Kansas Medical Center) ${ }^{21}$; and (3) mouse albumin was purchased from Sigma (St. Louis, MO, category number A1056). Protein purity was confirmed by SDS-polyacrylamide gel electrophoresis (Supplemental Figure 1, see http://ajp.amjpathol.org) and by Western blot with monoclonal antibodies anti Vsp1 (1H12) or anti Vsp2 (5F12) 20,22 (not shown). Lanthanide labeling of proteins with $\mathrm{Eu}$ or Sm was performed according to the manufacturer's instructions (Perkin-Elmer, Boston, MA). Briefly, $1 \mathrm{mg}$ of each protein resuspended in $50 \mathrm{mmol} / \mathrm{L} \mathrm{NaCO} 3 \mathrm{pH} 9.3$ was incubated with $\mathrm{Eu}$ or $\mathrm{Sm}$ labeling reagent (Eu-DTTA DELFIA Eu-labeling kit number 1244-302 or Sm-DTTA DELFIA Sm-labeling kit number 1244-303; Perkin-Elmer) at $25^{\circ} \mathrm{C}$ in the dark for 12 hours. The separation of lanthanide-labeled proteins from unbound lanthanides was done by column chromatography (Sephadex G-25 chromatography, Sigma 54805), which resulted in two peaks: the fist peak represents proteins and the second unbound Eu-chelate. To further minimize the contamination of lanthanide-labeled proteins with unbound lanthanidechelates, we dialyzed the solutions against distilled water by using $8000 \mathrm{Da}$ dialysis cassettes until the fluorescence of the exchange water was similar to that of water alone (Slide-A-Lyzer dialysis Cassette, category number 66380; Pierce/Thermo Fisher Scientific, Waltham, MA). For the lipidated proteins (LVsp1 and LVsp2), trace de- tergent was added to the water to prevent their precipitation. After dialysis, all lanthanide-labeled proteins were filtered to remove any remaining traces of unbound lanthanide chelate (Amicon Ultra15, number UFC901008; Millipore, Bedford, MA). The yield of Eu bound to protein in $(\mathrm{mol} / \mathrm{mol})$ ratio was $6.3: 1,5.64: 1,8.7: 1$, and 14:1 for LVsp1, LVsp2, rVsp1, and albumin, respectively; it was similar for all three $V s p$ proteins and higher for albumin, as expected based on its higher molecular weight.

Lanthanide labeling was confirmed by mass spectrometry in the protein core facility at the University of Medicine and Dentistry of New Jersey, New Jersey Medical School (courtesy of Hong Li, PhD). Modification with Europium was investigated by using a local MASCOT search engine (version 1.9) on a GPS (version 3.5, ABI) server. We confirm for Eu-rVsp1 that it did contain the Eu-label; the predicted molecular weight of the peptide SVKDVHTLVKSIDELAKAIGK had increased its predicted molecular mass from 2251.30 Da to $2733 \mathrm{Da}$, and the mass difference of $482.15 \mathrm{Da}$ was identical to that of the Eu-labeling reagent.

\section{Animal Model}

Groups of 5- to 7-week-old female $\mathrm{C} 3 \mathrm{H} / \mathrm{HeJ}$ or C57BL/6RAG1 deficient mice were obtained from Jackson Laboratories (Bar Harbor, ME) or Taconic Farms (Germantown, NY). For infection we inoculated $10^{3} \mathrm{~B}$. turicatae serotype 1 spirochetes intraperitoneally in $200 \mathrm{ml}$ of PBS or PBS alone as a control. To study lipoprotein dissemination, we used a dose of $100 \mu \mathrm{g} / \mathrm{kg}(\sim 2 \mu \mathrm{g}$ total dose per mouse), which represents approximately the amount of lipoprotein extracted from $10^{6} \mathrm{~B}$. turicatae spirochetes; pilot experiments revealed that up to $200 \mu \mathrm{g} / \mathrm{kg}$ of $\mathrm{li}$ poprotein could be given to a mouse without any obvious morbidity or mortality. The route of protein release into mice was intraperitoneal because preliminary studies showed that injection into the tail vein resulted in very rapid elimination of Eu-labeled proteins via the urine with little if any protein reaching tissues (data not shown). For all studies of protein dissemination, we injected mice intraperitoneally with $100 \mu \mathrm{g} / \mathrm{kg}$ of lanthanide (Eu or Sm) labeled proteins diluted in $200 \mu$ l of PBS; all necropsy days included two to three mice injected with PBS alone for measurement of time-resolved fluorescence (TRF) background signal. We repeated all experiments at least twice for consistency. The standard procedure was to necropsy five to seven protein-injected mice and two to three PBS injected mice each necropsy day. Mice were anesthetized with isoflurane before euthanasia by cardiac exsanguination. Plasma was obtained by brief centrifugation of heparinized blood. All mice had intracardiac total-body perfusion with $30 \mathrm{ml}$ of PBS to minimize blood contamination of the tissue intravascular compartment; adequate perfusion was confirmed by finding counts per second on the buffer coming out of the mouse heart at the end of the perfusion that were similar to those of buffer alone (not shown). All brains had to be free of macroscopic blood to be accepted for further processing; less than $10 \%$ of the brains had to be discarded because of residual blood contamination. 


\section{Protein Extraction and Measurement of Time Resolved Fluorescence}

We did protein extraction from fresh tissues and fluids harvested at necropsy. One hundred milligrams of each relevant tissue (brain, brain parenchymal, capillary fractions, lung, kidney, or liver), $100 \mu \mathrm{l}$ of plasma, or $\sim 50 \mu \mathrm{l}$ of urine were placed in lysing matrix tubes (QBiogene/MP Biomedicals, Solon, $\mathrm{OH}$; category number 6912), mixed with $1 \mathrm{ml}$ of protein extraction buffer (FastProtein red kit category, MP Biomedicals, number 6550-700), and homogenized in the FastPrep24 instrument (MP Biomedicals category number 6003500). Protein homogenates were kept overnight at $4^{\circ} \mathrm{C}$, and the next day centrifuged for 30 minutes at $3000 \mathrm{~g}$. The supernatant containing lanthanide labeled-protein in tissue or fluid protein extracts was used for testing by TRF. To release TRF from lanthanide-labeled protein, $1 \mu \mathrm{g}$ of each protein extract was diluted in $200 \mu \mathrm{l}$ of enhancing solution (Perkin Elmer, category number B118-100), incubated with agitation at room temperature for $\geq 20$ minutes, and centrifuged for 10 minutes at $3000 \mathrm{~g}$; 5 to 10 replicates of $200 \mu \mathrm{l}$ of each supernatant were place in 96-well micro plates (Delfia Yellow Plate, category number AAAND-0001; Perkin Elmer) and the counts per second per well measured by TRF by using a Victor 3 fluorometer (Perkin Elmer). ${ }^{23}$ The TRF program performs 100 pulses per second with an excitation light at $340 \mathrm{~nm}$ and an emission light at 615 $\mathrm{nm}$ for $\mathrm{Eu}$ or $640 \mathrm{~nm}$ for Sm. In the period between flashes, the sample is measured with a $400 \mu$ s delay; this eliminates interference from short-lived fluorescence, effectively reducing background fluorescence and increasing the sensitivity and specificity to levels comparable with isotopic labeling. ${ }^{24}$

\section{Brain Capillary Depletion}

For brain capillary depletion, we used dextran density centrifugation that depletes brain homogenate from its vasculature. ${ }^{25}$ For this, after intracardiac perfusion with buffer, we removed the brain, weighed it, and emulsified it with a syringe plug (10 strokes) in $0.8 \mathrm{ml}$ of physiological buffer (10 $\mathrm{mmol} / \mathrm{L}$ HEPES, $141 \mathrm{mmol} / \mathrm{L} \mathrm{NaCl}, 4 \mathrm{mmol} / \mathrm{L} \mathrm{KCl}, 2.8$ $\mathrm{mmol} / \mathrm{L} \mathrm{CaCl} 2,1 \mathrm{mmol} / \mathrm{L} \mathrm{MgSO}{ }_{4}, 1 \mathrm{mmol} / \mathrm{L} \mathrm{NaH} \mathrm{PO}_{4}$, and $10 \mathrm{mmol} / \mathrm{L} \mathrm{d}$-glucose) adjusted to $\mathrm{pH}$ 7.4. Dextran solution ( $1.6 \mathrm{ml}$ of a $26 \%$ solution) was added to the homogenate, which was vortexed and homogenized again (three strokes). Homogenization was performed at $4^{\circ} \mathrm{C}$ in less than 1 minute. An aliquot of the homogenate was centrifuged at $5400 \mathrm{~g}$ for 15 minutes. The pellet containing the brain parenchyma and the supernatant containing the capillaries were carefully separated and processed for protein extraction as described above.

\section{Stability of Eu-Labeled Proteins in Vivo}

To measure the stability of Eu-labeled proteins in vivo, we compared the dissemination from peritoneum to plasma and excretion in the urine of Eu-labeled proteins with that of the Eu-chelate alone. For this we injected groups of mice ( $N=4$ to 5 each) intraperitoneally with $10^{7} \mathrm{cps}$ of
Eu-chelate alone or Eu-labeled proteins and measured counts per second in $10 \mu \mathrm{l}$ of plasma and urine 2, 4, 12, and 18 hours after inoculation. The results showed that the kinetics of dissemination to the plasma and excretion in the urine were different between the Eu-labeled proteins and the Eu-chelate alone (Supplemental Figure 2, see $h$ ttp://ajp.amjpathol.org).

\section{Protein Dissemination in Vivo}

To measure the dissemination of lanthanide-labeled proteins from the peritoneum to urine, plasma, or tissues, we used ratios comparing the counts per second per microliter of plasma or urine or per microgram of tissue protein extracts relative to the total counts per second in the original intraperitoneal inoculum after subtracting the average background counts per second with the following formula: (cps TE or PE - cps TC or PC)/total cps of inoculum) $\times 1000 \mathrm{~g}$, where $T$ refers to tissue, $P$ to plasma, $E$ to mice inoculated with lanthanide-labeled proteins, and $\mathrm{C}$ to mice sham-inoculated with PBS as fluorescence background controls. We used plasma rather than whole blood because preliminary experiments showed that in whole blood the proteins were distributed in the plasma rather than the red blood cell pellet (not shown). The distribution of proteins from plasma to tissues was compared by ratios of counts per second per microgram of protein in plasma to tissue with the following formula: [(cps TE/cps PE)/(cps TC/cps PC)] × 100, where T refers to tissue, $P$ to plasma, $E$ to mice inoculated with lanthanide-labeled proteins, and C to mice sham-inoculated with PBS as fluorescence background controls. The crossing of lanthanide-labeled proteins across the BBB was similarly calculated by using ratios with the following formula: [(cps BFE/cps PE)/(cps BFC/cps PC)] $\times 100$, where $\mathrm{BF}$ refers to brain fraction (either parenchymal or capillary), $\mathrm{P}$ to plasma, E to mice inoculated with lanthanide-labeled proteins, and $\mathrm{C}$ to mice sham-inoculated with buffer as fluorescence background controls.

\section{DNA Extraction and PCR}

DNA was extracted from $100 \mathrm{mg}$ of whole brain or brain parenchymal or capillary fractions and from $100 \mu$ l of necropsy plasma pellets by using the QIAamp DNA Micro kit (Qiagen, Inc., Turnberry, CA) followed by elution in $50 \mu \mathrm{l}$ Tris-EDTA buffer $\mathrm{pH} 7.4$ and storage at $-80^{\circ} \mathrm{C}$. TaqMan quantitative PCR reactions used $100 \mathrm{ng}$ of whole brain/brain fraction DNA or $10 \mathrm{ng}$ of plasma pellet DNA. TaqMan assays were run in multiplex, with predesigned mouse 18S rRNA primers and probe set as host housekeeping gene (Mm4308329; Applied Biosystems, Foster City, CA) and a $16 \mathrm{~S}$ borrelial ribosomal primers and probe set ${ }^{4,5}$ in the same well. Quantitative real time PCR was performed on an ABI Prism 7500 Sequence Detection System (Applied Biosystems) in $50 \mu \mathrm{l}$ of reaction volumes with TaqMan Universal master mix reagents (Applied Biosystems) under standard conditions for 40 cycles. A standard curve using $\log _{10}$ dilutions from DNA extracted from a known number of cultured Bt1 spirochetes in $100 \mathrm{ng}$ of uninfected host DNA was used for 
absolute quantification. Analysis was done with the $A B \mid$ Sequence Detection System software version 1.2.3 by extrapolation of the sample's threshold cycle to the standard curve. Bt1 dissemination ratios were calculated by using the following formula: number of borrelias in $100 \mathrm{ng}$ of plasma DNA/number of borrelia in $100 \mathrm{ng}$ of brain DNA, $\times 100$

\section{RNA Extraction and RT-PCR}

Total RNA was extracted from $100 \mathrm{mg}$ of whole brain or brain fraction homogenates (FastPrep24 homogenizer, MP Biomedicals) by using the phenol/chloroform method followed by DNase treatment (Ambion Kit, Applied Biosystems). RNA was quantified with a nanodrop by using A260/A280 ratios. One microgram of RNA per sample was reversed transcribed with random hexamers by using the Transcriptor First Strand cDNA Synthesis Kit (Roche Applied Science, Penberg, Germany) in $20 \mu \mathrm{l}$ reaction volumes. Quantitative real time PCR was performed on an ABI Prism 7500 Sequence Detection System (Applied Biosystems), in multiplex, by using 10\% of each cDNA in $50 \mu$ l reaction volumes with TaqMan Universal master mix (Applied Biosystems) and standard conditions for 40 cycles. Mouse 18S rRNA (4319413E, Applied Biosystems) was used as endogenous control for input brain RNA. The primers and probe for interleukin (IL)-10 (Mm00439616_m1), F4/80 (Mm00802529_m1), and CXCL13 (Mm01208154- g1) were from Applied Biosystems. Analysis was performed by using 1.2.3 AB Sequence Detection System software. For relative quantification of mRNA expression, the $\Delta \Delta \mathrm{Ct}$ method was applied ${ }^{26}$ by using a single calibrator from an uninfected mouse brain for all assays. Results were analyzed adjusting for the amount of input cDNA by using the mouse chromosomal gene for $18 S$ rRNA and expressed as the $\log 2$ negative numbers $\left(2^{-} \Delta \Delta \mathrm{Ct}\right)$; therefore, higher values represent higher gene copy number relative to the target gene.

\section{Statistical Analysis}

All statistical analyses were performed by using GraphPad PRISM 4 (GraphPad Software). All data are presented as mean (SD), median (range), or box plots. Oneway (Kruskal-Wallis test with Dunn's test for multiple comparisons) or two-way analysis of variance (Bonferroni posttest for multiple comparisons) were used to compare data depending on the distribution. Spearman's test was used to determine correlations. $P<0.05$ was considered significant, $P<0.01$ or $P<0.001$ was considered highly or very highly significant, respectively.

\section{Results}

\section{LVsp1 Disseminates to the Brain from a Distant Site}

We began the study by comparing the dissemination of Eu-labeled proteins to plasma and urine after their re-
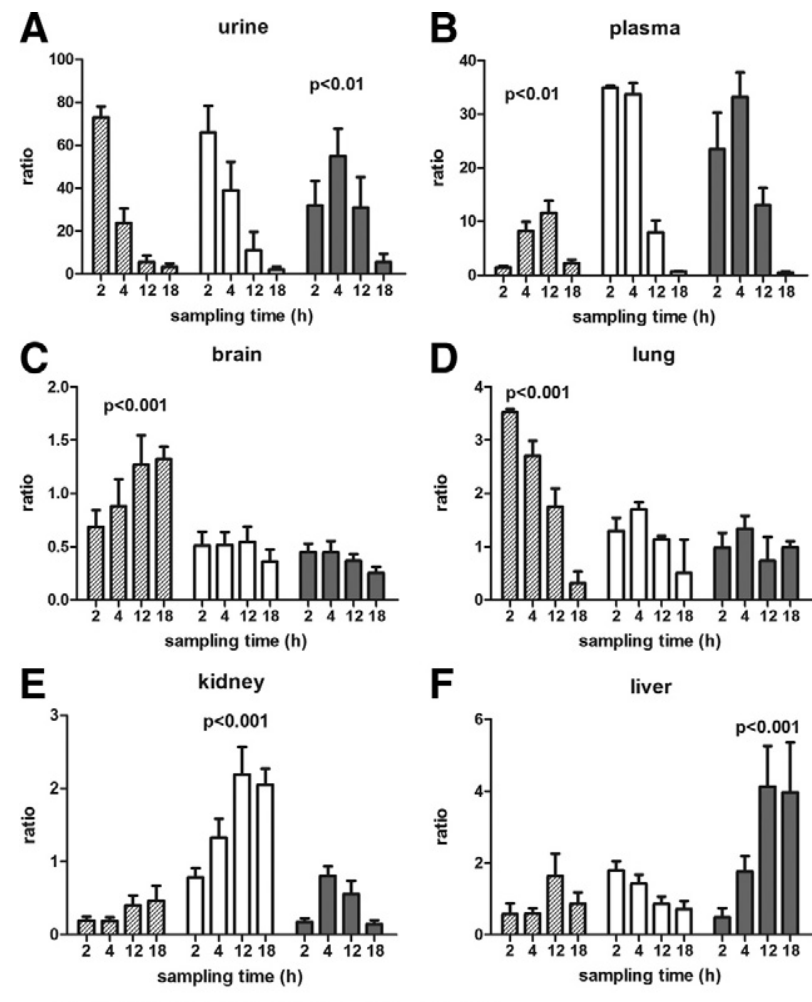

$\mathbf{F}$

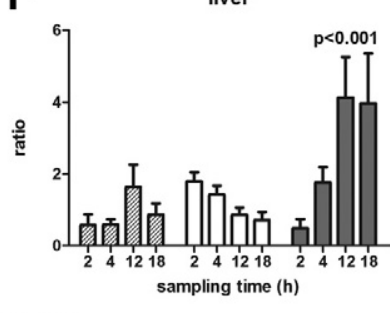

Eu-LVsp1 $\square$ Eu-rVsp1 $\square$ Eu-albumin

Figure 1. Dissemination of Eu-labeled proteins. A and B: Dissemination kinetics from peritoneum to plasma and urine 2, 4, 12, and 18 hours after i.p. injection of $10^{7}$ counts per second of each Eu-labeled protein $(n=5$ mice each). C-F: Dissemination kinetics from peritoneum to tissues 2, 4, 12, and 18 hours after i.p. injection with $100 \mu \mathrm{g} / \mathrm{kg}$ of each Eu-labeled protein $(n=$ 5 mice each). The bars represent mean (SD) of two separate experiments. $P$ values were calculated by two-way analysis of variance and show the tissue distribution for each Eu-labeled protein. Notice that LVsp1 shows preferential dissemination from the peritoneum to the brain and lung.

lease into the peritoneum. For this we injected $10^{7} \mathrm{cps}$ of Eu-LVsp1, Eu-nonlipidated Vsp1 (Eu-rVsp1), or Eumouse albumin (Eu-albumin) into groups of $\mathrm{C} 3 \mathrm{H} / \mathrm{HeJ}$ mice and collected blood and urine over several hours for examination by TRF. The results showed distinct differences in the kinetics of dissemination between the three proteins: although dissemination to the urine was faster for the two Vsp1 proteins than for albumin (Figure 1A, $P<$ 0.01 at 2 to 12 hours), dissemination to plasma (Figure 1B) was slower for Eu-LVsp1 compared with Eu-rVsp1 and Eu-albumin $(P<0.001$ for the comparisons at 2 and 4 hours). Next we studied whether there were differences in the dissemination of the three proteins to the brain and other organs. For this we injected new groups of $\mathrm{C} 3 \mathrm{H} /$ HeJ mice with $100 \mu \mathrm{g} / \mathrm{kg}$ of Eu-labeled proteins intraperitoneally and measured their dissemination by using ratios of counts per second per microgram of tissue protein to counts per second in the original inoculum. Extensive perfusion with buffer was used to minimize blood contamination of tissues. The results revealed marked differences in the dissemination of the three proteins to organs (Figure 1, C-F): although Eu-LVsp1 showed preferential dissemination to the brain (Figure 1C) and the lung (Figure 1D), Eu-rVsp1 showed preferential dissemination to the kidney and Eu-albumin to the liver $(P<0.001$ for all). 
A

A
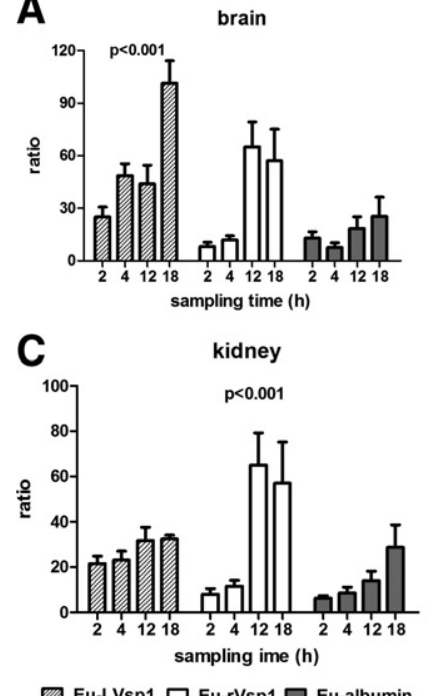

B

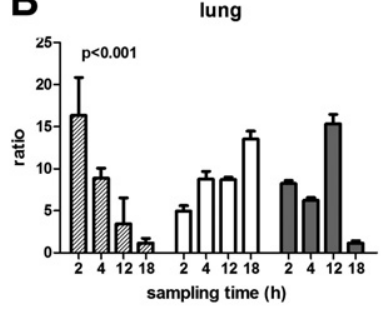

D

liver

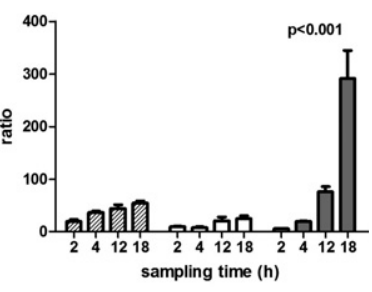

Figure 2. Protein dissemination from blood to tissues: brain (A), lung (B), kidney (C), and liver (D). Groups of five mice each were necropsied 2, 4, 12, or 18 hours after i.p. injection with $100 \mu \mathrm{g} / \mathrm{kg}$ of each Eu-labeled protein. The bars represent mean (SD) of two separate experiments. $P$ values were calculated by two-way analysis of variance and show the distribution from plasma to tissue over time for each Eu-labeled protein. Notice that LVsp1 shows preferential distribution from plasma to the brain.

Interestingly, progressive accumulation of Eu-LVsp1 occurred in the brain but not in the lung. Next we measured whether there were similar differences in the distribution of the three proteins between plasma and organs over time. For this we used ratios of counts per second per microgram of tissue extracts relative to plasma. The results showed better distribution from plasma to brain and lung with Eu-LVsp1, to kidney with Eu-rVsp1, and to liver with Eu-albumin (Figure 2, A-D, all $P<0.001$ ). The distribution of Eu-LVsp1 from plasma to brain increased over time, whereas it decreased over time from plasma to lung (Figure 2A). We confirmed that the preferential dissemination of LVsp1 to the brain occurred independently of the lanthanide label used (Supplemental Figure 3A, see http://ajp.amjpathol.org) or the host's genetic background (Supplemental Figure 3B, see http://ajp.amjpathol.org). We concluded that labeled-LVsp1 disseminated from the periphery to the brain better than nonlipidated Vsp1 or mouse albumin.

\section{Bacterial Infection Increases the Dissemination of LVsp1 into the Brain}

Next we investigated the effect of infection on the dissemination of LVsp1 to the brain. We used two different models of bacterial infection by using Bt1, relapsing infection in $\mathrm{C} 3 \mathrm{H} / \mathrm{HeJ}$ mice and persistent infection in C56BL/6-RAG1 deficient (RAG1 ${ }^{-/-}$) mice. Groups of Bt1infected mice were injected intraperitoneally with 100 $\mu \mathrm{g} / \mathrm{kg}$ of Eu-labeled LVsp1, rVsp1, or albumin to study protein dissemination to the brain over time by using ratios as before. The results showed that both relapsing and persistent bacterial infection increased the dissemination of Eu-LVsp1 from peritoneum to brain (Figure 3A)

A peritoneum to brain
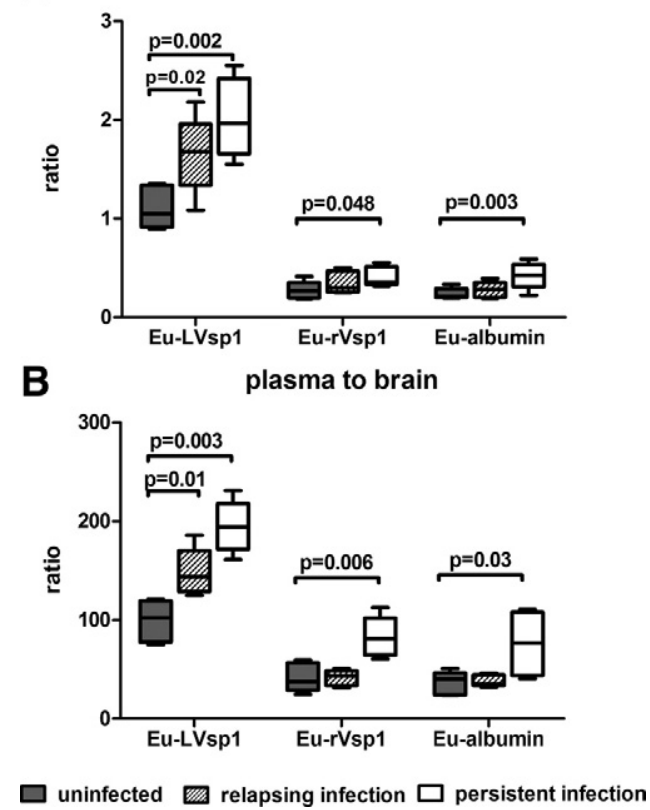

Figure 3. Effect of infection on the dissemination of LVsp1 from the periphery to the brain. Dissemination ratios of peritoneum to the brain (A) and plasma to the brain (B) 18 hours after i.p. injection with $100 \mu \mathrm{g} / \mathrm{kg}$ of each Eu-labeled protein into uninfected $\mathrm{C} 3 \mathrm{H} / \mathrm{HeJ}$ mice or relapsing $(\mathrm{C} 3 \mathrm{H} / \mathrm{HeJ})$ or persistently (C57BL/6-RAG1 deficient) Bt1-infected mice ( $n=5$ each). The results are presented as box plots for two to three separate experiments for each mouse model. $P$ values were determined by using the Kruskal-Wallis test. Notice that Eu-LVsp1 shows the greatest dissemination to the brain of all of the proteins tested. Also notice that either relapsing or persistent infection increased the dissemination of Eu-LVsp1 to the brain, whereas this occurred to a lesser extent with Eu-rVsp1 and Eu-albumin.

and its distribution from plasma to brain (Figure 3B). The increase in dissemination to the brain was higher with persistent than with relapsing infection $(P<0.01)$. Infection also increased the dissemination to brain of Eu-rVsp1 and Eu-albumin but to a lesser extent than Eu-LVsp1 and only if it was persistent (Figure 3, A and B). We concluded that bacterial infection increases the dissemination of LVsp1 from the periphery to the brain.

Next we investigated if the increased dissemination of LVsp1 to the brain during infection was the result of increased permeability of the BBB. For this we injected intraperitoneally new groups of uninfected or Bt1-infected $\mathrm{RAG}^{-1-}$ mice simultaneously with $100 \mu \mathrm{g} / \mathrm{kg}$ of Eu-albumin and $100 \mu \mathrm{g} / \mathrm{kg}$ of Sm-LVsp1 to determine whether the presence of Eu-LVsp1 increased the dissemination to the brain of Eu-albumin, which has a molecular weight three times larger than that of LVsp1. ${ }^{21}$ The results showed that the dissemination of Eu-albumin to brain did not change in the presence of Eu-LVsp1 (Supplemental Figure 4, see http://ajp.amjpathol.org; $P=N S$ ). We concluded that the increase in dissemination of LVsp1 to the brain as a result of infection was unlikely to be explained by nonspecific opening of the BBB.

\section{The Dissemination of $L V s p 1$ to the Brain Involves Crossing of the BBB}

Next we investigated whether the observed dissemination of Eu-LVsp1 to the brain represented intravascular 
A

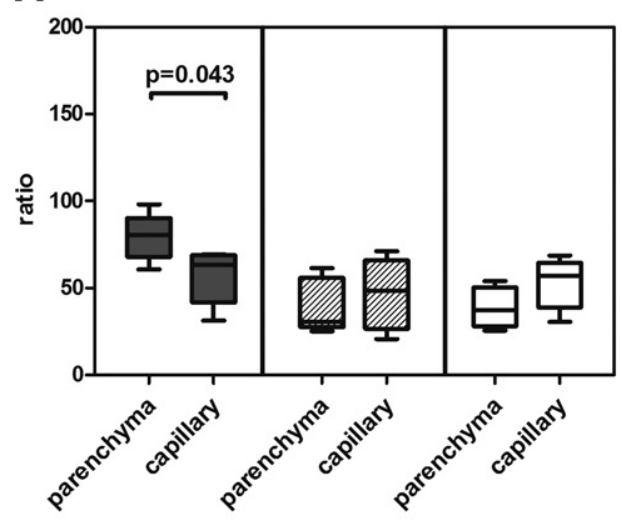

B

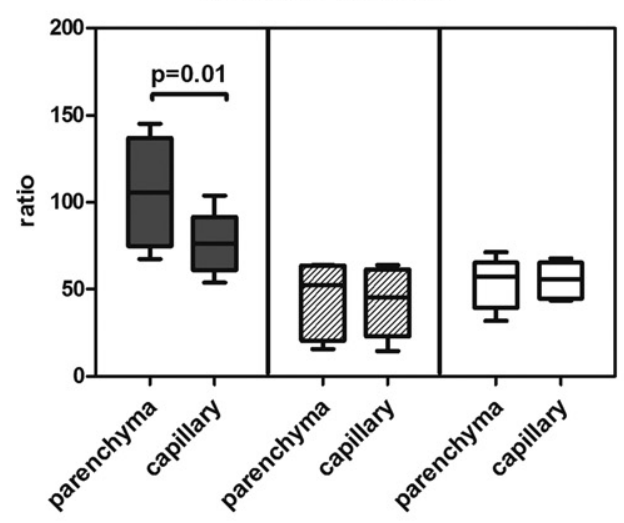

C persistent infection

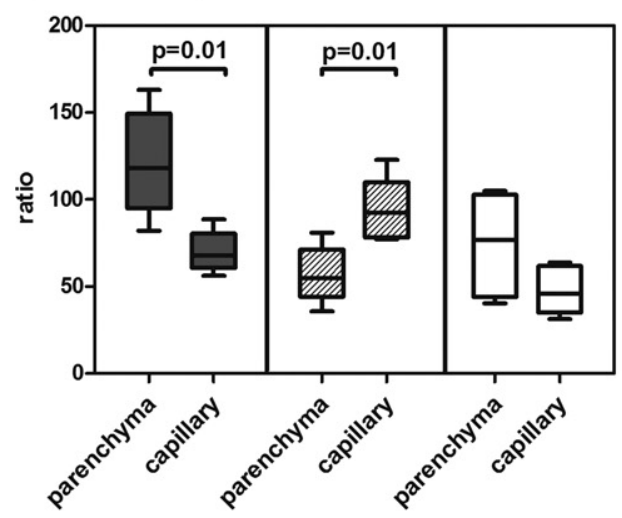

$\square$ Eu-LVsp1 四 Eu-rVsp1 $\square$ Eu-albumin

Figure 4. Dissemination of LVsp1 to the brain represents crossing of the blood-brain barrier. Dissemination from plasma to brain fractions 18 hours after i.p. injection with $100 \mu \mathrm{g} / \mathrm{kg}$ of each Eu-labeled protein in uninfected mice (A) or mice with relapsing (B) or persistent $(\mathbf{C})$ Bt1-infection. Mice with relapsing infection were $\mathrm{C} 3 \mathrm{H} / \mathrm{HeJ}$ necropsied on day five after inoculation of $\mathrm{Bt} 1$, whereas mice with persistent infection were C57BL/6-RAG1 deficient necropsied 12 days after inoculation with $\mathrm{Bt} 1$. The results are presented as box plots for two to three separate experiments for each model. $P$ values were calculated by using the Kruskal-Wallis test. Notice that LVsp1 is significantly increased in the brain parenchymal fraction compared with the brain capillary fraction in uninfected (A) and relapsing (B) or persistently (C) infected mice.

localization in brain capillaries and/or crossing of the BBB into the brain parenchyma. To measure BBB crossing, we used brain capillary depletion, an established method to determine whether proteins are taken up by the brain parenchyma as opposed to sequestered in the capillary bed. ${ }^{25}$ For this we measured dissemination to whole brain and brain fractions 4 and 18 hours after intraperitoneal injection of $100 \mu \mathrm{g} / \mathrm{kg}$ of Eu-labeled proteins in both uninfected mice and mice with relapsing or persistent infection. The results showed that Eu-LVsp1 showed preferential localization to the brain parenchymal fraction rather than the brain capillary fraction in all three models (Figure 4, A-C). This was not seen for Eu-albumin in any of the models. Eu-rVsp1 showed preferential localization to the brain capillary fraction but only during persistent infection (Figure 4C). We concluded that the observed dissemination of LVsp1 into the brain corresponded to preferential localization to the brain parenchymal fraction, consistent with BBB crossing into the central nervous system (CNS)

\section{The Dissemination of LVsp1 into the Brain \\ Parenchyma Causes Up-Regulation of Pro-Inflammatory and Anti-Inflammatory Mediators}

Next we studied whether the dissemination of LVsp1 to the brain parenchyma causes brain inflammation. For this we measured transcription of three genes that we have shown before are significantly up-regulated in the brain during infection with Bt1: the membrane glycoprotein of microglia/macrophages $\mathrm{F} 4 / 80,{ }^{3,4}$ the cytokine $\mathrm{IL}-10,{ }^{27,28}$ and the $\mathrm{B}$ cell chemokine CXCL13. 4,29,30 For this groups of uninfected $\mathrm{C} 3 \mathrm{H} / \mathrm{HeJ}$ mice were injected intraperitoneally with $100 \mu \mathrm{g} / \mathrm{kg}$ of Eu-LVsp1 or Eu-albumin, and changes in gene transcription were measured 18 hours later by using TaqMan RT-PCR. The results showed upregulation in the brain of all three genes examined with Eu-LVsp1 compared with Eu-albumin: the fold difference was 1.5 for $\mathrm{F} 4 / 80(P=0.02)$, 1.9 for $\mathrm{CXCL} 13(P=0.048)$, and 4.9 for $\mathrm{IL}-10(P=0.01)$. A comparison between protein dissemination to the brain and up-regulation of pro-inflammatory and anti-inflammatory genes revealed strong positive correlations for Eu-LVsp1 (Figure 5, A-C). The strongest correlation, $r=0.81$, was with $\mathrm{IL}-10$, an anti-inflammatory cytokine that plays critical protective roles during infection with relapsing fever spirochetes in humans and experimental animals. 4,5,28,31,32 We concluded that the dissemination of LVsp1 into the brain parenchyma induces both inflammatory and anti-inflammatory reactions. The results also suggested stronger induction of anti-inflammatory (IL-10) than of pro-inflammatory (CXCL13) molecules.

\section{LVsp1 Disseminates to the Brain Parenchyma Better Than Bt1 and LVsp2}

The original observation that triggered the current study was that we could not explain the widespread cerebra microgliosis observed in mice persistently infected with Bt1 in B cell deficient mice by the presence of spirochetes in the brain parenchyma. ${ }^{1}$ Therefore, next we compared the ability of LVsp1 and Bt1 to disseminate 
A

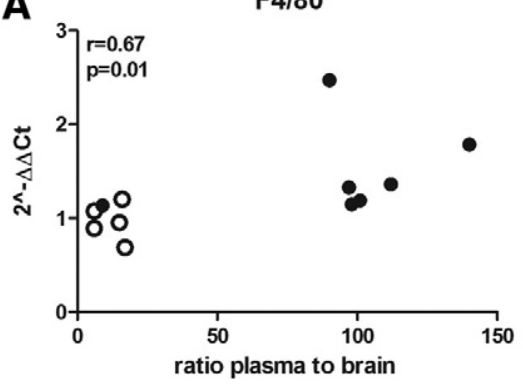

B

CXCL13

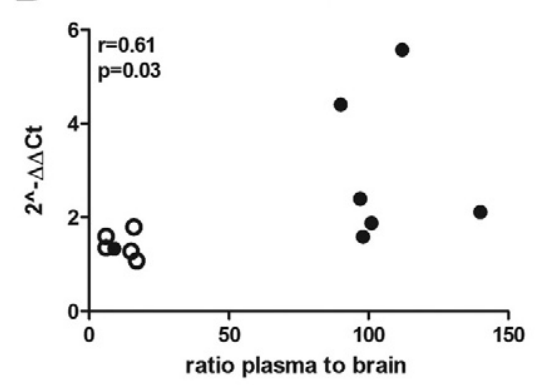

C

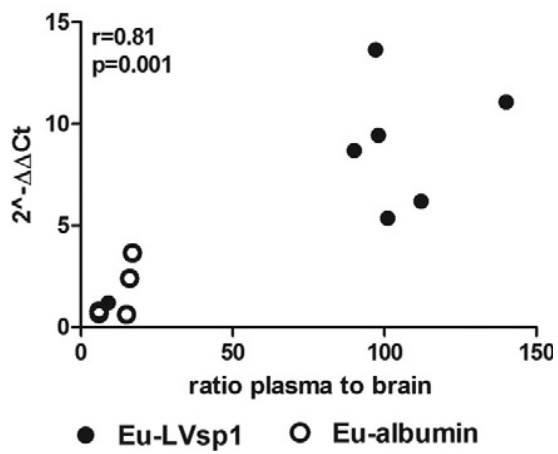

Figure 5. Entry of LVsp1 into the brain parenchyma causes up-regulation of brain pro-inflammatory and anti-inflammatory mediators. Correlation between brain gene expression with ratios of dissemination of Eu-labeled proteins from plasma to brain 18 hours after i.p. injection with $100 \mu \mathrm{g} / \mathrm{kg}$ of either Eu-LVsp1 $(n=7)$ or Eu-albumin $(n=5)$ into uninfected C3H/HeJ mice. Correlation and $P$ values were determinate by Spearman's test. Notice a positive correlation between the entry of Eu-LVsp1 into the brain parenchyma and up-regulation of F4/80 (A), CXCL13 (B), and IL-10 (C). This was not observed with the Eu-albumin used as control.

from blood to brain parenchyma. Groups of RAG $1^{-1-}$ mice persistently infected with Bt1 for 2 weeks were injected intraperitoneally with $100 \mu \mathrm{g} / \mathrm{kg}$ of Eu-LVsp1 and 4 or 18 hours later necropsied to compare the distribution of Eu-LVsp1 and Bt1 DNA between blood and brain. Bt1 DNA was measured by TaqMan RT-PCR amplification of the chromosomal gene $16 S$ rRNA. The results in the mice necropsied 4 hours after protein injection showed that Eu-LVsp1 had disseminated to whole brain and brain parenchymal fraction significantly better than Bt1 (Figure 6A). The distribution from plasma to brain parenchymal fraction was fourfold higher for Eu-LVsp1 than for Bt1 DNA $(P=0.002)$ even though mice had been infected with Bt1 for 2 weeks and injected with Eu-LVsp1 only 4 hours earlier. Mice necropsied 18 hours after protein injection showed that the distribution of Eu-LVsp1 from blood to brain had continued to increase compared with

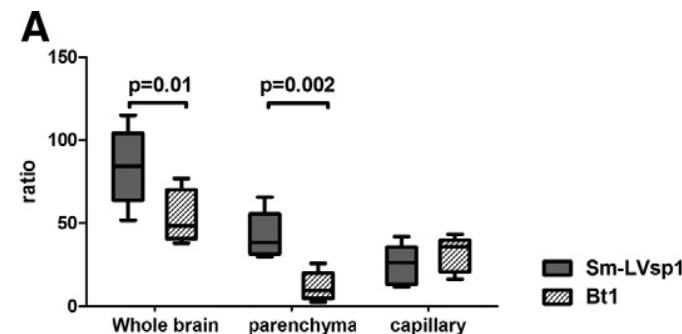

B

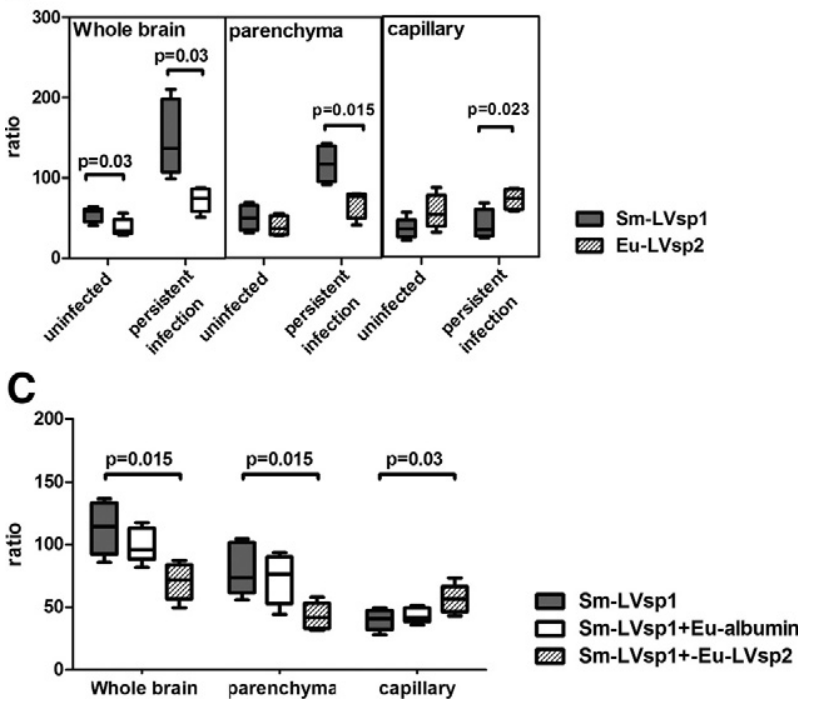

Figure 6. A: LVsp1 disseminates into the brain parenchyma better than Bt1. Ratios of distribution of Eu-LVsp1 and Bt1 spirochetes from blood to brain four hours after i.p. injection with $100 \mu \mathrm{g} / \mathrm{kg}$ of Eu-LVsp1 to C57BL/6-RAG1 deficient mice that had been infected for two weeks $(n=7)$. Bt1 was measured by TaqMan PCR amplification of the borrelial gene $16 \mathrm{~S} r R N A$. Results show box plots for two separate experiments each. $P$ values were calculated by using Mann-Whitney tests. Notice preferential distribution of Eu-LVsp1 into the brain parenchymal fraction, whereas Bt1 spirochetes preferentially distributed into the brain capillary fraction. B: LVsp1 disseminates from the periphery into the brain better than LVsp2. Dissemination ratios of plasma to whole brain or brain fraction four hours after i.p. injection with $100 \mu \mathrm{g} / \mathrm{kg}$ of Sm-LVsp1 or Eu-LVsp2 into groups of uninfected mice or mice persistently infected with Bt1 ( $n=6$ each). Results are presented as box plots for two separate experiments for each model. Notice that LVsp1 disseminated to whole brain and brain parenchymal fraction better than LVsp2 C: LVsp2 reduces the dissemination of LVsp1 into the brain parenchyma. Dissemination ratios from plasma to whole brain or brain fraction four hours after i.p. injection with Sm-LVsp1 alone $(100 \mu \mathrm{g} / \mathrm{kg})$ or in combination with Eu-LVsp2 or Eu-albumin ( $100 \mu \mathrm{g} / \mathrm{kg}$ of each) into groups of uninfected mice ( $n=6$ each). Results are presented as box plots for two separate experiments for each model. $P$ values were determined by using the Kruskal-Wallis test. Notice that simultaneous injection with Eu-LVsp2, but not with Eu-albumin, reduced the dissemination of Sm-LVsp1 from plasma to brain parenchyma.

mice necropsied at 4 hours, whereas that of Bt1 remained the same (not shown). We concluded that LVsp1 disseminated into brain parenchyma better than the spirochete from which it comes from, Bt1.

Next we studied whether LVsp1 disseminated to the brain better than a similar bacterial lipoproteins. For this we compared it with LVsp2, the outer membrane lipoproteins of isogenic Bt2 that has the same lipidation but is $32 \%$ different from LVsp1 at the amino acid level, ${ }^{22}$ mostly in the variable Vsp dome region. ${ }^{33}$ We chose to compare LVsp1 with LVsp2 because during persistent infection Bt 1 causes 5 to 10 times greater brain infection and inflammation than Bt2. 2,3,18 New groups of uninfected and persistently infected mice were injected intra- 
peritoneally with $100 \mu \mathrm{g} / \mathrm{kg}$ of Sm-LVsp1 or Eu-LVsp2 and necropsied after 4 or 18 hours to compare protein dissemination from blood to brain. The results at 4 hours showed that Sm-LVsp1 showed greater dissemination to whole brain and the brain parenchymal fraction than Eu-LVsp2 in both uninfected and persistently infected mice (Figure 6B). At 18 hours the median (range) ratio of dissemination to the brain parenchymal fraction was 184 (274-133) for Sm-LVsp1 compared with 94 (161-170) for LVsp2 $(P<0.05)$. The corresponding values for the capillary-enriched brain fraction were 107 (155-185) for Sm-LVsp1 and 169 (222-130) for Eu-LVsp2 $(P<0.05)$. Persistent infection increased dissemination to the brain with both proteins but to a larger extent with Sm-LVsp1 than with Eu-LVsp2. Sm-LVsp1 showed preferential localization to the brain parenchymal fraction, whereas EuLVsp2 showed preferential localization to the brain capillary fraction (Figure 6B). We concluded that LVsp1 distributed from blood to brain better than LVsp2.

Because both lipoproteins share the same lipidation but differ in their variable region at the Vsp dome, ${ }^{33}$ we tested whether the dissemination of LVsp1 into the brain is affected by the presence of LVsp2. For this we injected new groups of uninfected C3H/HeJ mice with $100 \mu \mathrm{g} / \mathrm{kg}$ of Sm-LVsp1 alone or in combination with $100 \mu \mathrm{g} / \mathrm{kg}$ of Eu-LVsp2 or $100 \mu \mathrm{g} / \mathrm{kg}$ of Eu-albumin. Mice were necropsied 4 hours later to measure protein dissemination. The results showed that the dissemination of Sm-LVsp1 to the brain decreased in the presence of Eu-LVsp2 but not in the presence of Eu-albumin (Figure 6C). The localization of Sm-LVsp1 to the brain capillary fraction increased in the presence of Eu-LVsp2 (Figure 6C). Similar results were obtained in mice persistently infected with Bt1: Sm-LVsp1 preferentially localized to the brain parenchymal fraction when injected alone $(P=0.039)$ but not when injected together with Eu-LVsp2 $(P=N S$; not shown). We concluded that the presence of LVsp2 shifts the localization of LVsp1 in the brain from the parenchymal to the capillary fraction. This suggests that both the lipidation and conserved structural features of the Vsp protein family are important for brain dissemination.

Finally, we compared the dissemination of LVsp1 and LVsp2 from peritoneum to blood, urine, and tissues other than the brain. For this we injected new groups of uninfected C3H/HeJ mice intraperitoneally with $100 \mu \mathrm{g} / \mathrm{kg}$ of Sm-LVsp1 or Eu-LVsp2 and measured their distribution. The results showed similar dissemination from peritoneum to plasma and urine for both proteins but greater dissemination of Eu-LVsp2 from peritoneum to liver and kidney $(P<0.01$ for both) and from plasma to liver and kidney $(P<0.001$ for both; Figure $7, A-C)$. We concluded that LVsp2 disseminated to liver and kidney better than LVsp1.

\section{Discussion}

The intriguing observation of widespread cerebral microgliosis in mice persistently infected with Bt 1 that could not be explained by the presence of spirochetes in the brain parenchyma was the motivation for the current study. We

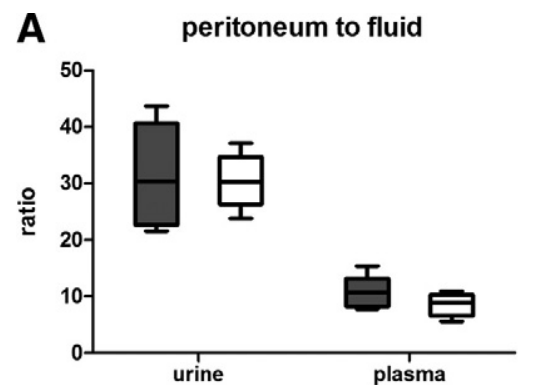

B peritoneum to tissue
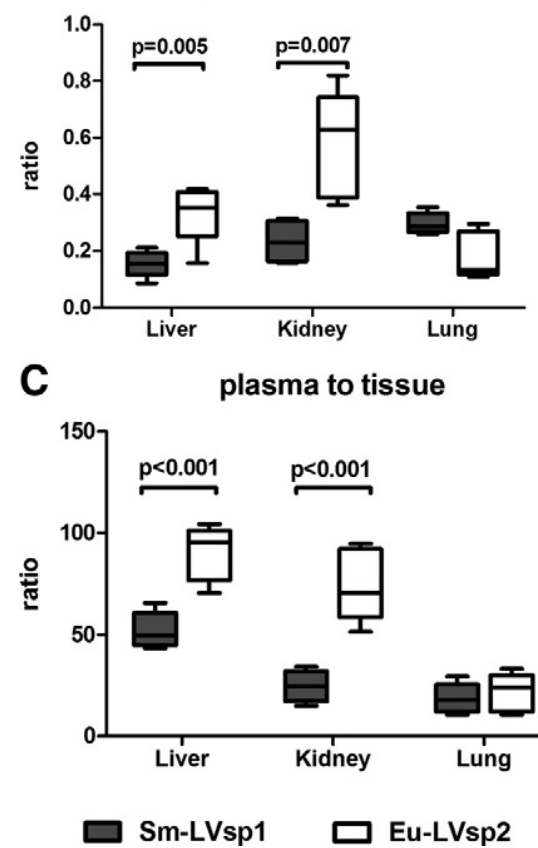

Figure 7. Differences in dissemination of LVsp1 and LVsp2 to blood, urine and tissues other than the brain. Dissemination ratios from peritoneum to plasma and urine $(\mathbf{A})$ or tissues $(\mathbf{B})$ and from plasma to tissues $(\mathbf{C}) 4$ hours after i.p. injection with $100 \mu \mathrm{g} / \mathrm{kg}$ of Sm-LVsp1 or Eu-LVsp2 $(n=5$ each). The results are presented as box plots of two separate experiments, and $P$ values were calculated by using the Kruskal-Wallis test. Note the higher dissemination for LVsp2 to liver and kidney compared with LVsp1.

hypothesized that pro-inflammatory bacterial products released from abundant spirochetes in the periphery could explain the observed cerebral inflammation if they were capable of disseminating to the brain parenchyma. Here we studied this possibility by measuring the dissemination of LVsp1 to the brain parenchyma after injection into the peritoneum. The major findings are the following: (1) LVsp1 can disseminate to the brain from a distal site; (2) the dissemination of LVsp1 into the brain represents crossing of the BBB into the brain parenchyma; (3) the dissemination of LVsp1 across the BBB results in induction of pro-inflammatory and anti-inflammatory responses; (4) the lipid modification and structural restricted to Vsp1 are involved in the dissemination of LVsp1 into the brain parenchyma; (5) infection increases the dissemination of LVsp1 into the brain parenchyma; (6) LVsp1 disseminates to the brain parenchyma better than the spirochete where it comes from, Bt1; and (7) LVsp1 disseminates to the brain better than LVsp2, whereas 
LVsp2 disseminates to liver and kidney better than LVsp1.

Since the initial experiments, it became evident that LVsp1 was capable of dissemination to the brain from the periphery (Figures 1 and 2). All subsequent experiments were supportive of this: LVsp1 dissemination to the brain occurred independently of the lanthanide-label we used for tracing (Supplemental Figure 3A, see http://ajp. amjpathol.org) and the genetic background of the mice (Supplemental Figure 3B, see http://ajp.amjpathol.org). Furthermore, although brain dissemination occurred under normal conditions, it greatly increased in the presence of relapsing-remitting and persistent infection. The finding that in the brain LVsp1 preferentially localizes to the brain parenchymal rather than the brain capillary fraction indicates that it actually crosses the BBB rather than simply binding to or internalizing into brain endothelial cells. Because LVsp1 entry into the brain parenchyma did not increase BBB crossing of mouse albumin (Supplemental Figure 4, see http://ajp.amjpathol.org)., we do not believe that BBB crossing of LVsp1 is due to nonspecific opening of the tight junctions. In fact, the finding that brain entry of LVsp1 was greatly reduced by the loss of the lipidation, as in rVsp1, a change in the variable protein region, as in LVsp2, or by the presence of LVsp2, points to a complex mechanisms in which the presence of the lipidation as well as both conserved and variable structural features of the protein are involved.

Previously we found that surface proteolysis eliminates the binding of amino acid-radiolabeled Bt1 spirochetes to human brain microvascular endothelial cells (HBMEC). ${ }^{3}$ More recently we found that purified Eu-LVsp1 binds to and enters HBMEC in vitro, a process in which the lipid modification plays an essential role (Diana Londono, Gaurav Gandhi et al, manuscript in preparation). Interestingly, the association of Eu-LVsp1 with HBMEC was inhibited by 10-fold excess amounts of unlabeled LVsp1 or LVsp2 but not by rVsp1 or albumin (Diana Londono, Gaurav Gandhi et al, manuscript in preparation). These observations are consistent with the findings from the current study in which the absence of the lipid modification and the presence of LVsp2 interfered with BBB crossing by LVsp1. Altogether these findings support a model in which the successful interaction of LVsp1 with brain endothelial cells that results in CNS penetration is dependent on the presence of the lipid modification as well as conserved and variable Vsp structural features. ${ }^{33}$ Because surface proteolysis of HBMEC completely eliminated the association of $\mathrm{Bt} 1$ with $\mathrm{HBMEC}^{3}$ it is likely that the mechanism of LVsp1 entry into the CNS involves receptor-specific mediated transport across brain endothelial cells into the brain parenchyma. This putative LVsp1 receptor on brain endothelial cells could be a cell surface protein that triggers membrane transport followed by release of LVsp1 at the abluminal side. The alternative possibility is that LVsp1 causes nonspecific opening of the BBB similar to LPS ${ }^{11}$; as discussed, this appears less likely in view of the finding that co-injection of LVsp1 with albumin did not result in increased entry of albumin into the CNS (Supplemental Figure 4, see http://ajp.amjpathol.org).
Surprisingly little is known about how bacterial products in the periphery can affect the brain. One of the best studied is LPS, which is responsible for the pathophysiological changes associated with Gram-negative infections, including the prominent neurological dysfunction of sepsis. Earlier studies revealed accumulation of LPS into tissues, including the brain, after peripheral injection of toxic doses. ${ }^{34}$ However, subsequent studies showed that LPS does not cross the BBB but rather binds to receptors in brain endothelial cells and circumventricular organs. ${ }^{11}$ Studies with the gp120 coating protein of HIV-1 have shown that it can cross the BBB by adsorptive endocytosis. ${ }^{35,36}$ Sialic and $\mathrm{N}$-acetyl- $\beta$-D-glucosaminyl acids are involved in brain endothelial cell uptake of gp120. ${ }^{37}$ Blood-borne horseradish peroxidase has also been shown to be endocytosed avidly at the luminal face of the cerebral endothelium. ${ }^{38}$ Studies of whether human lipoproteins can cross the BBB have shown that although acetylated low density lipoprotein is rapidly bound by the brain microvasculature after intra-arterial injection, it does not appear to undergo transport into the brain parenchyma. $^{25}$

The neuropathology of $B$. turicatae infected mice is dominated by cerebral microgliosis without any detectable injury. ${ }^{3,4,28,31} \mathrm{~A}$ prominent product of activated microglia in mice infected with $B$. turicatae without brain injury is $\mathrm{IL}-10,{ }^{27}$ which suggested it is neuroprotective. This was recently confirmed by the rapid morbidity and mortality from intracerebral and subarachnoid brain hemorrhage observed in $B$. turicatae infected mice deficient in IL-10.6,28,32 Intraperitoneal injection of LVsp1 or LVsp2 induces systemic production of IL-10 (D. Londoño, D. Cadavid, unpublished results. This is similar to what is observed in experimental animals and humans infected with relapsing fever spirochetes. ${ }^{4,5,27,32,39}$ This suggests that the mammalian host responds to the presence of borrelia lipoproteins with preferential production of IL-10 both systemically and locally, which appears to prevent vascular injury. In this regard it is interesting that dissemination of LVsp1 into the brain parenchyma resulted in strong up-regulation of IL-10, far greater than that of than of F4/80 or CXCL13 (Figure 5).

In conclusion, the finding that LVsp1 disseminates to the brain parenchyma after intraperitoneal injection provides proof of principle that during systemic bacterial infections brain inflammation can be caused by dissemination of pro-inflammatory products released locally or in the circulation to brain. It will be important to determine whether this phenomenon occurs with other infectious agents and in human disease.

\section{Acknowledgment}

We thank Dr. Allen C. Steere (Center of Immunology and Inflammatory Diseases, Massachusetts General Hospital) for his support and for critically reviewing the article. 


\section{References}

1. Cadavid D, Pachner AR, Estanislao L, Patalapati R, Barbour AG: Isogenic serotypes of Borrelia turicatae show different localization in the brain and skin of mice. Infect Immun 2001, 69:3389-3397

2. Cadavid D, Sondey M, Garcia E, Lawson CL: Residual brain infection in relapsing-fever borreliosis. J Infect Dis 2006, 193:1451-1458

3. Sethi N, Sondey M, Bai Y, Kim KS, Cadavid D: Interaction of a neurotropic strain of Borrelia turicatae with the cerebral microcirculation system. Infect Immun 2006, 74:6408-6418

4. Gelderblom H, Londono D, Bai Y, Cabral ES, Quandt J, Hornung R, Martin R, Marques A, Cadavid D: High production of CXCL13 in blood and brain during persistent infection with the relapsing fever spirochete Borrelia turicatae. J Neuropathol Exp Neurol 2007, 66:208-217

5. Londono D, Marques A, Hornung RL, Cadavid D: IL-10 helps control pathogen load during high-level bacteremia. J Immunol 2008, 181:2076-2083

6. Cluss RG, Silverman DA, Stafford TR: Extracellular secretion of the Borrelia burgdorferi Oms28 porin and Bgp, a glycosaminoglycan binding protein. Infect Immun 2004, 72:6279-6286

7. Barbour AG, Todd WJ, Stoenner HG: Action of penicillin on Borrelia hermsii. Antimicrob Agents Chemother 1982, 21:823-829

8. Garon CF, Dorward DW, Corwin MD: Structural features of Borrelia burgdorferi: the Lyme disease spirochete; silver staining for nucleic acids. Scanning Microsc Suppl 1989, 3:109-115

9. Sadziene A, Jonsson M, Bergstrom S, Bright RK, Kennedy RC, Barbour AG: A bactericidal antibody to Borrelia burgdorferi is directed against a variable region of the OspB protein. Infect Immun 1994, 62:2037-2045

10. Huang SH, Jong AY: Cellular mechanisms of microbial proteins contributing to invasion of the blood-brain barrier. Cell Microbiol 2001, 3:277-287

11. Singh AK, Jiang Y: How does peripheral lipopolysaccharide induce gene expression in the brain of rats? Toxicology 2004, 201:197-207

12. Haake DA: Spirochaetal lipoproteins and pathogenesis. Microbiology 2000, 146(pt 7):1491-1504

13. Brandt ME, Riley BS, Radolf JD, Norgard MV: Immunogenic integral membrane proteins of Borrelia burgdorferi are lipoproteins. Infect Immun 1990, 58:983-991

14. Sambri V, Cevenini R: Incorporation of cysteine by Borrelia burgdorferi and Borrelia hermsii. Can J Microbiol 1992, 38:1016-1021

15. Weis JJ, Ma Y, Erdile LF: Biological activities of native and recombinant Borrelia burgdorferi outer surface protein $A$ : dependence on lipid modification. Infect Immun 1994, 62:4632-4636

16. Erdile LF, Brandt MA, Warakomski DJ, Westrack GJ, Sadziene A, Barbour AG, Mays JP: Role of attached lipid in immunogenicity of Borrelia burgdorferi OspA. Infect Immun 1993, 61:81-90

17. Hirschfeld M, Kirschning CJ, Schwandner R, Wesche H, Weis $\mathrm{JH}$, Wooten RM, Weis JJ: Cutting edge: inflammatory signaling by Borrelia burgdorferi lipoproteins is mediated by toll-like receptor 2. J Immunol 1999, 163:2382-2386

18. Cadavid D, Thomas DD, Crawley R, Barbour AG: Variability of a bacterial surface protein and disease expression in a possible mouse model of systemic Lyme borreliosis. J Exp Med 1994, 179:631-642

19. Ivey NS, Martin EN Jr, Scheld WM, Nathan BR: A new method for measuring blood-brain barrier permeability demonstrated with Europium-bound albumin during experimental lipopolysaccharide (LPS) induced meningitis in the rat. J Neurosci Methods 2005, 142:91-95

20. Cadavid D, Pennington PM, Kerentseva TA, Bergstrom S, Barbour AG: Immunologic and genetic analyses of $\mathrm{VmpA}$ of a neurotropic strain of Borrelia turicatae. Infect Immun 1997, 65:3352-3360
21. Zuckert WR, Kerentseva TA, Lawson CL, Barbour AG: Structural conservation of neurotropism-associated VspA within the variable Borrelia Vsp-OspC lipoprotein family. J Biol Chem 2001, 276:457463

22. Pennington PM, Cadavid D, Barbour AG: Characterization of VspB of Borrelia turicatae, a major outer membrane protein expressed in blood and tissues of mice. Infect Immun 1999, 67:4637-4645

23. Dickson EF, Pollak A, Diamandis EP: Time-resolved detection of lanthanide luminescence for ultrasensitive bioanalytical assays. J Photochem Photobiol B 1995, 27:3-19

24. Jackson TM, Ekins RP: Theoretical limitations on immunoassay sensitivity: current practice and potential advantages of fluorescent Eu3+ chelates as non-radioisotopic tracers. J Immunol Methods 1986, 87:13-20

25. Triguero D, Buciak J, Pardridge WM: Capillary depletion method for quantification of blood-brain barrier transport of circulating peptides and plasma proteins. J Neurochem 1990, 54:1882-1888

26. Livak KJ, Schmittgen TD: Analysis of relative gene expression data using real-time quantitative PCR and the 2(-Delta Delta $\mathrm{C}(\mathrm{T})$ ) method. Methods 2001, 25:402-408

27. Gelderblom H, Schmidt J, Londono D, Bai Y, Quandt J, Hornung R, Marques A, Martin R, Cadavid D: Role of interleukin 10 during persistent infection with the relapsing fever Spirochete Borrelia turicatae. Am J Pathol 2007, 170:251-262

28. Londono D, Carvajal J, Arguelles-Grande C, Marques A, Cadavid D: Interleukin 10 protects the brain microcirculation from spirochetal injury. J Neuropathol Exp Neurol 2008, 67:976-983

29. Cadavid D: The mammalian host response to borrelia infection. Wien Klin Wochenschr 2006, 118:653-658

30. Narayan K, Dail D, Li L, Cadavid D, Amrute S, Fitzgerald-Bocarsly P, Pachner AR: The nervous system as ectopic germinal center: CXCL13 and IgG in lyme neuroborreliosis. Ann Neurol 2005, $57: 813-823$

31. Cadavid D, Londono D: Understanding tropism and immunopathological mechanisms of relapsing fever spirochaetes. Clin Microbiol Infect 2009, 15:415-421

32. Londono D, Marques A, Hornung RL, Cadavid D: Relapsing fever borreliosis in IL-10 deficient mice. Infect Immun 2008, 76:5508-5513

33. Mehra R, Londono D, Sondey M, Lawson C, Cadavid D: Structurefunction investigation of vsp serotypes of the spirochete Borrelia hermsii. PLoS One 2009, 4:e7597

34. Musson RA, Morrison DC, Ulevitch RJ: Distribution of endotoxin (lipopolysaccharide) in the tissues of lipopolysaccharide-responsive and -unresponsive mice. Infect Immun 1978, 21:448-457

35. Broadwell RD, Balin BJ, Salcman M: Transcytotic pathway for bloodborne protein through the blood-brain barrier. Proc Natl Acad Sci USA 1988, 85:632-636

36. Villegas JC, Broadwell RD: Transcytosis of protein through the mammalian cerebral epithelium and endothelium. II. Adsorptive transcytosis of WGA-HRP and the blood-brain and brain-blood barriers. J Neurocytol 1993, 22:67-80

37. Banks WA, Kastin AJ: Characterization of lectin-mediated brain uptake of HIV-1 GP120. J Neurosci Res 1998, 54:522-529

38. Balin BJ, Broadwell RD, Salcman M, el-Kalliny M: Avenues for entry of peripherally administered protein to the central nervous system in mouse, rat, and squirrel monkey. J Comp Neurol 1986, 251:260-280

39. Cooper PJ, Fekade D, Remick DG, Grint P, Wherry J, Griffin GE: Recombinant human interleukin-10 fails to alter proinflammatory cytokine production or physiologic changes associated with the Jarisch-Herxheimer reaction. J Infect Dis 2000, 181:203-209 